International Journal of Behavioral Research \& Psychology (IJBRP)

ISSN 2332-3000

\title{
Beyond Organic Aetiology: Exploring A Psychocognitive Approach To Gastric Ulceration
}

Review Article

\section{Michael O.S. Afolabi ${ }^{1 *}$}

${ }^{1 *}$ Centre for Healthcare Ethics,Duquesne University, Pittsburgh, P.A., U.S.A.

\begin{abstract}
Pavlov's classical conditioning experiments brought to the fore how stimuli of purely psychogenic nature mediate and affect cognitive state, hence, shaping physiologic functions. Against this background, I seek to understand how the triad of the brain, the mind and our human experiences (in the context of disease) interact. I attempt a systematic explication of how this interaction may occur in the onset of gastric ulceration. On that note, I argue that a valid psychocognitive frame of reference offers creative insights into how non-pharmacological means may be employed in the clinical palliation of forms of non-organic gastric ulcers.
\end{abstract}

Key Words: Gastric Ulceration; Thought; Classical Conditioning.

\section{*Corresponding Author:}

Michael O.S. Afolabi,

Centre for Healthcare Ethics,Duquesne University, Pittsburgh, P.A., U.S.A.

E-mail: curiousmaikl@yahoo.com

Received: July 22, 2013

Accepted: August 08, 2013

Published: August 09, 2013

Citation: Michael O.S. Afolabi (2013)Beyond Organic Aetiology: Exploring A Psychocognitive Approach To Gastric Ulceration. Int J Behav Res Psychol. 1(1), 1-4. doi: https://dx.doi.org/10.19070/2332-3000130001

Copyright: Michael O.S. Afolabi ${ }^{\circ}$ 2013. This is an open-access article distributed under the terms of the Creative Commons Attribution License, which permits unrestricted use, distribution and reproduction in any medium, provided the original author and source are credited.

\section{Introduction}

Ivan Pavlov's[1] classic experiments on conditioned reflexes as well as the subsequent theory of classical conditioning they engendered highlighted how carefully associating a novel stimulus with a 'regular' stimulus can come to substitute for the latter in mediating physiologic functions. That is, the selective dependence of certain physiologic functions upon context-defined stimuli. Hence, the fascinating experience of dogs exhibiting salivation upon associating bell-ringing with food availability. In modern thought, classical conditioning has been found to be a widespread phenomenon even in humans, its potential for occurring being high in our day-to-day experiences[2].

While classical conditioning has been known to occur in humans since the time of Pavlov [3], it has been hitherto explicated in a manner determined by purely external stimuli. In this essay, I argue that applied to humans, and under a given set of conditions; the Pavlovian notion may be extended to the realm of the mind as a region of stimuli production, association and, hence, a region where conditioning can occur. In other words, I advance the thesis that intrinsic stimuli from within may establish classical conditioning. On this note, I attempt to forge a conceptual nexus around the triad of the brain, the mind and our human experiences in the context the possible onset of gastric ulceration.

Indeed, if infection with Helicobacter pylori is neither sufficient nor necessary for the development of gastric ulcer disease [4], and if gastric ulceration has still been observed in patients who do not demonstrate significant overuse of non-steroidal anti-inflammatory drugs (NSAIDS); it is only proper to commence locating the aetiological agents in previously unexplained cases elsewhere. It may be noted that previous attempts to look elsewhere in relation to the organic aetiology of gastric ulceration have focused on stress. Stress, in this sense, conveys the idea of a physical or psychological stimulus (occupational overwork, bereavement, divorce et cetera) which alters the homeostatic mechanisms of the body, generating a 'load' which may either be handled, and, when not, lead to disease. In relation to gastric ulceration, psychophysiological studies have shown chronic psychological stress to be associated with hypersecretion of gastric hydrochloric acid, which falls upon abatement of the stressful state [7]. The stress idea is however not central to the idea raised in this paper.

\section{Methods \& Goals}

This essay employs the method of archival research. It attempts a systematic harmonization of notions derived from varied academic disciplines such as physiology, gastroenterology, and neurobiology. It offers an analytic examination of how the mind may influence the disease experience in the onset of gastric ulceration. Against this template, it seeks creative insights into the feasibility of employing psychocognitive means for the clinical palliation of forms of gastric ulceration that have been hard to explain with recourse to organic aetiology.

\section{Conceptual Clarifications}

A number of clarifications are of imperative before this essay can properly begin. Gastric and peptic/duodenal ulcers have been described as ulcerative lesions of the stomach and duodenum respectively [7]. But because gastric and peptic ulcers are not reliably 
differentiated by either history or physical signs [8], I shall employ the term 'gastric ulceration' in an all-inclusive manner to describe both forms of ulceration. In the world-view of medicine as in most medical sciences, the dominant notion of the human body is embedded in the machine metaphor. This notion conceives the human body as a self-regulating 'machine' who's teleological functions are ingrained in ensuring survival via maintenance of both internal and external homeostasis. On this premise, behavioral patterns are assumed to stem from the brain in a one-to-one relationship. Hence, the mind constitutes a mere appendage or "a shadow of the physiological brain and disappears when the latter disappears" [5].

It is hardly surprising therefore that most health professionals take the position that the mind and body are not separate entities, but are a part of an interacting whole [24]. Yet, this is only partly true; for we are confronted with the age-long Cartesian dualism or mind-body problem. While philosophical ideas about the mind, brain and behavior often seem theoretical and unimportant to those involved in the medical sciences [6], this is actually an interesting debate. Research on split-brain patients has, for instance, raised questions about the implications of split-brain surgery to the mind-brain problem. The argument goes that if the surgeon's knife is able to separate consciousness, then splitting the brain is an equivalent of splitting the mind. But it has been difficult (if not impossible) to validate the claim that such patients possess two realms of consciousness [19]. To be sure, since the human brain lacks certain properties exclusively associated with the mind [6]; the mind and the biological brain should be considered as distinct from each other.

The human brain is therefore conceived as the part of the central nervous system encased in the cranium and comprised of a complex assortment of neural networks. The mind denotes not only the human seat of preconsciousness and subconsciousness but also the non-material 'region' of the body where individual qualities such as ambition, decision-making and willpower reside, and from where they are exercised to govern and regulate behavior. It is taken for granted that the latter capacity may be mediated in synergy with specialized parts of the brain as dictated by the nature of the activity in question. Against this backdrop, this paper subsumes 'thoughts', 'habits of thoughts' and 'tendencies of thought patterns' under the regulation of the mind. Having made these clarifications, the paper begins with a brief remark about the physiology of gastric secretion in relation to gastric ulceration.

\section{The Mind Vis-à-vis Gastric Secretion}

Physiologists have since delineated the phases involved in the process of gastric secretion as well as their inducing signals/stimuli. These are the cephalic, the gastric and the intestinal phases in their order of expression. While each of these phases contributes its part to the total volume of juice secreted, it may be noted that the cephalic (brain) phase is responsible for about $20 \%$ of this volume [9]. It is indeed interesting to note that the cephalic phase of gastric secretion occurs in response to such signals as the sight, smell (aroma, in social parlance) and thoughts of food. In other words, pre-feeding thinking of food even in the absence of the actual meal/food as well as in the absence of the aroma triggers neurogenic signals either in the cerebral cortex, amygdala, or the hypothalamus [9], a physiological event which transmits neural signals to the left and right branches of the vagi. The vagi, in turn, cause the parietal (oxyntic) and G cells in the stomach walls to secrete hydrochloric acid and proteolytic enzymes via the action of histamine[8],[10]. It has been said that the mind makes adaptive behavior possible [26]; the adaptive value of thought-induced or pre-feeding secretion of gastric acid may therefore be that it prepares the systems of the body involved in the digestion of macromolecules ahead of the actual process of digestion, and may help the system function in a coordinated and integrated fashion.

Inhelder and Piaget posit the idea that children hardly think about their thoughts as they lack the power of systematic reflection [11] Obviously, this notion may be retrospectively applied to babies. Hence, no thought-induced gastric secretion can be said to occur in children and babies. One can however assume (from an evolutionary viewpoint) that babies have a physiological sensor capable of detecting low blood glucose. Upon detecting glucose level fall below a given threshold, this sensor would trigger a crying response from the lower brain centers such as the amygdala, which ultimately attracts the mother's attention; thus, bringing about the suckling act which eventually restores the glucose level and equally abates the crying. Heath has argued that no new evolutionary structure (hence, function) has appeared in the human species in the last 3,000 years [12]. The explanatory model offered by the glucoserestoring framework would therefore persist in the young as in the adult human, though undergoing some modifications based on learning and adaptation, a process which closely involves the brain, and made possible by its 'rewireable' or plastic nature. That is, how the brain's neural networks change with experience[13].

For children, this adaptation comes via the process of 'culinary socialization' in which their feeding patterns are tailored along clearly defined lines (e.g. on a three meal per day basis). Since the culinary habit of adults hardly fall within such a well defined framework, it is possible to attempt an explanation of how thought-induced gastric secretion in adults under a given set of conditions may be the aetiological agent in a number of hitherto unexplained cases of gastric ulcerative lesions. That is, in those $5 \%-20 \%$ of adult patients in whom no clear organic etiologic agent have been implicated [4]. Critical to accomplishing that task however is a conceptual elaboration of what is meant by the term 'thought'.

Thought has been described as trial behavior; that is, activities in the making. Behavior itself has been linked with metabolic processes [12]. At a level of analysis, it can be said that changes in the metabolic configuration of certain parts of the nervous system can initiate the thinking process. This framework fits into the holistic notion of thought which holds that thought results from multiple concomitant stimulation of many parts of the nervous system, an event involving "the cerebral cortex, thalamus, limbic system and the upper reticular formation of the brain stem"[9] But how could this scene be possibly played out in the process of gastric secretion? That is, how may we possibly explicate the intricate underlying connection between thoughts about food in relation to gastric ulceration? It is instructive to chart and explore what actually mediates the 'thought' that causes gastric secretion.

'Thought about food' may be a spontaneously generated neural signal in response to the glucose sensor ${ }^{\mathrm{i}}$ which registers upon the template of the appetite centers of the hypothalamus in the brain, from where these are channeled to the cerebral cortex, which houses the associative cortex, and is concerned with intelligence and problem solving [14]. The adult human species on having this impression registered upon his/her mind, and depending on the environmental context in which the impression is made, then 
'decides' on whether to embark on eating or not. Let us call this the evolutionarily mediated gastric secretion (EMGS). To be sure, the adult human engages the realm of cognition to modify the empirical world[11], a pragmatic manner through which this occurs in the context of feeding is through establishing a somewhat normative value around his eating patterns.

Thinking bears close correlation to introspection, a process that describes attending to the content of one's consciousness [15]. On the other hand, if human behavior is indeed a consequence of the activity of the mind [12]; at another level of analysis, it may be said that thought-induced gastric secretion ensues from conscious expenditure of thought in the direction of food. Since mentation involves reality conceived in specific ways, in certain ways rather than others, and may be about what may not be in the immediacy of the environment [6], conscious thinking about food may be undertaken with and without the presence of food. That is, whether such thoughts are predicated upon physiological needs or an individually created desire for eating. Yet, the physiological consequence of each, in terms of gastric secretion, would be same. Let us call the latter idiosyncratically mediated gastric secretion (IMGS). Weisz describes how a similar phenomenon occurs in the context of having a conversation over the phone [27], a process which not only brings the mind of the other alive in the ambit of imagination but ensures that the whole gamut of responses that would have been produced in actual proximity are elaborated.

Lastly, it has been noted that many human goals extend beyond any given situation, and that these goals need to be maintained in the mind of the individual probably in an unconscious form [16]. It follows therefore that putting a conscious halt to a thought does not necessarily halt it at a subconscious level. Hence, ending thoughts about food may not translate into its eradication at the level of the subconscious. Hence, food thoughts entertained at the plane of sub consciousness would likewise bring about gastric secretion. Let us call this the subconsciously mediated gastric secretion (SMGS). While this notion raises the notion of whether or not stimuli of which one is unaware might influence behavior [17], it has been observed that introspection or thinking affects and influences preconscious as well as post perceptual processes [18]. Hence, subconscious activity can shape human behavior.

With these possible clarifications of how different manners of entertaining thoughts about food translate into the physiological event of gastric secretion, the next part of this paper goes into the conceptual interface of probing how the Pavlovian theory of classical conditioning may offer useful explanations vis-à-vis the onset of some gastric ulceration due to non-organic causes.

\section{Thought-Induced Gastric Ulceration Vis-à-vis Classical Conditioning}

In the Pavlovian experiment on classical conditioning, there was an unconditioned stimulus, US, which mediated an unconditioned response, UR. There also was a conditioned stimulus, CS, which mediated a conditioned response, CR. Further, once a CS predicts a US a CR will develop. On this note, we may characterize food as the US which mediates the UR of gastric secretion. 'Food thoughts' would then constitute the CS which imitates and brings about the CR of gastric secretion.

However, thoughts about food, as have been shown, can bring about gastric secretion at three possible levels (that is, EMGS,
IMGS and SMGS). Examined and interpreted through an evolutionary lens, it follows that IMGS and SMGS result from an aspect of classical conditioning referred to as generalization. It is important to further state that generalization describes a new conditioned response that occurs to an established CS when the new CS resembles the initial CS to which a response has been previously conditioned [3]. Now, where does all this lead us in relation to the thesis that certain patterns of thoughts may help explain forms of gastric ulceration which have been explained by organic aetiologies?

Man is a complex being, this complex nature being a determinant of the multifarious behavioral patterns that he exhibits. It is therefore only natural to expect that aside from the EMGS there could be other forms of thought-induced gastric secretion. Given this scheme of things, the SMGS appears to be the aetiological agent for hitherto unexplained causes of gastric ulceration. This brings us to the idea of reinforcement. Reinforcement is an essential feature in the manifestation of classical conditioning [28], the absence of which leads to extinction; that is, a break in the CS-US relation. Indeed, without reinforcement there occurs an associational deficit [29].

Given that humans are more inclined to be motivated to seek activities which have a rewarding aura [30], the nature of the IMGS would hardly meet the requirements for establishing reinforcement for the CR. A possible and obvious objection which may be leveled against the idea that subconscious stimuli about food bring about gastric ulceration might be raised via the idea of habituation. Habituation is the decrease in response to repeated stimulation mediated in the central nervous system [31]. However, while habituation to external stimuli leads to decreased response, internally mediated stimuli are known to produce continuous responses.

Indeed, if human consciousness is indeed a recently evolved feature [19], subconscious activities would more or less be exerted from so-called lower brain centers such as the amygdala. This makes some sense, because the amygdala is closely associated with non-declarative memory which does not require conscious awareness and is expressed without conscious effort20 indeed, nondeclarative memory has been found to be linked with classical conditioning21. While almost every one of us would from time to time fit into the SMGS framework, it may be that some persons have an exaggerated pattern or habit of thought in relation to food which would make them more susceptible. Expressed differently, it may be that persons whose attitudinal response to the satiation of the 'hunger reflex' is pursued in a more fervent manner would exhibit the SMGS higher than those whose attitudes are not as fervent. In light of the individual differences in physiological reactivity, it is possible also that some individuals merely respond to the SMGS in an exaggerated manner. While these possibilities may explain why the basal gastric acid level in patients with gastric ulceration is often higher than normal [22], they likewise offer a model for explaining the cause, hence, source of the acidic onslaught which in time erodes the gastrointestinal mucosa, and brings about the manifestation of ulcers.

\section{Conclusions}

There is room for rational speculation in science [23]. Subjected to results obtained from the laboratory of empirical/experimental validation, such a speculation undergoes slight or varied reconfigurations which ultimately increase its scope and predictive 
power, thus, enriching our understanding of biological or phenomenological realities. This essay should therefore be seen as a conceptual excursion. The demonstration of its pragmatic and clinical merit requires experimental investigation. Whatever the specific answers, a theory and praxis built around the interaction of thought patterns on the brain, and its role in gastric ulceration offers creative insights in the sphere of rendering a non-pharmacologic intervention to the disease. This non-pharmacologic window would be found in breaking the CS — CR association through appropriate psychocognitive means. The thesis advanced in this essay may, indeed, help explicate why gastric ulceration is not so common in children.

\section{References}

[1]. Pavlov, Ivan P. (1927) Conditioned Reflexes Oxford: Oxford University Press.

[2]. Crider, A.B, Goethals, G.R. and Kavanaugh, R. (1983) Psychology Glenview: Scott, Foreman and Company, p. 195.

[3]. McSweeney, F. and Bierley C. (1984) Recent Developments in Classical Conditioning Journal of Consumer Research 11, 2; 619-631.

[4]. Jones, Michael J (2006) The Role of Psychosocial Factors in Peptic Ulcer Disease: Beyond Helicobacter pylori and NSAIDS Journal of Psychosomatic Research 60, 407-412.

[5]. Smith, Wilder A. E. (1970) The Drug Users: the Psychopharmacology of Turning on Illinois: Harold Shaw Publishers, p. 140.

[6]. Bolton, D. and Hill, J. (1996) Mind, Meaning and Mental Disorder Oxford University Press, pp. vii, 2-3, 19, 36, 38, 73

[7]. Bennett, P. and Douglas, C. (1997) Gastric and Duodenal Ulcers in: Cambridge Handbook of Psychiatry, Health and Medicine (Eds.) Baum, A., Stanton, N., Weinman, J. et al, Cambridge University Press, pp. 464-465.

[8]. Brunt, P. W., Losowsky, M. S. and Read A. E (1988) Gastroenterology London: Heinemann Medical Books pp. 102, 111

[9]. Guyton, A. and Hall, J.E. (2000) Textbook of Human Physiology Philadelphia: W B. Saunders Company, Tenth Edition.

[10]. Davies, A., Asa, G.H.B. and Cecil, K. (2001) Human Physiology Edinburgh: Churchill Livingstone, $\mathrm{p} 827$

[11]. Inhelder, B. and Piaget J. (1958) The Growth of Logical Thinking from Childhood to Adolescence New York: Basic Books, pp. 339, 346.

[12]. Heath, Robert G. (1996) Explaining the Mind-Brain Relationship Baton Rouge: Moran Publishing Inc. 8-9, 33, 39, 59, 157

[13]. McEwen, Bruce (1999) Stress and the Brain in: States of Mind (Ed.) Roberta Conlan New York: John Wiley and Sons Inc, p. 94.

[14]. Lenzer, Irmingard I (1979) Brain and Behaviour: Recent Topics in Human Psychophysiology in: Brain, Behaviour and Environmental Psychology (Eds.) Lenzer, I.I, Chadwick J.K, Darley, J. and Hill, K.A. Baltimore: University Park Press p18
[15]. Overgaard, Morten (2006) Introspection in Science Cognition and Consciousness 15,$4 ; 629-633$.

[16]. Smallwood, J., Rory C.O. and Megan, V.S. et al (2005) The Consequences of Encoding Information on the Maintenance of Internally Generated thoughts: the Role of Meaning Complexes Cognition and Consciousness 13, 4; 789-820.

[17]. Kiesel, A., Annika, W., Wilfried K. et al (2006) Unconscious Manipulation of Free choice in Humans Consciousness and Cognition 15, 2; 397-408.

[18]. Overgaard, M., Mika, K., Thomas A.S., et al (2006) The Electrophysiology of Introspection Cognition and Consciousness 15, 4; 662 - 672.

[19]. Springer, S.P. and Deutsch, G. (1989) Left Brain, Right Brain New York: W. H. Freeman and Company, pp. 322, 326.

[20]. Kandel, Eric (1999) Of Learning, Memory and Genetic Switches in: State of Mind (Ed.) Roberta Conlan New York: John Wiley and Sons Inc, p. 166.

[21]. Clark, R.E. and Squire, C.R. (1998) Classical Conditioning and Brain Systems: the Role of Awareness Science 280, 5360; 77-81.

[22]. Jones, P.F., Brunt, P.W. and Mowat, A.G.H (1985) Gastroenterology London: Heinemann Medical Books Ltd, p. 76.

[23]. Rhodes, Philip (1976) The Value of Medicine London: George Alan \& Unwin Ltd; p. 36.

[24]. Gatchel, Robert J (1997) Psychophysiology in: Cambridge Handbook of Psychiatry, Health and Medicine (Eds.).) Baum, A., Stanton, N., Weinman, J. et al, Cambridge University Press, pp. 45, 48.

[25]. Patrick, G.T.W. (1922) The Emergent Theory of Mind the Journal of Philosophy 19, 26; 701-708.

[26]. Weisz, Judit Szekacs (2007) Mindless Bodies-Bodyless Minds The American Journal of Psychoanalysis 67, 291-298. 10

[27]. Razran, Gregory (1955) Operant vs. Classical Conditioning American Journal of Psychology 68, 3; 489-490.

[28]. Schmajuk, N.A. and Larrauri, J.A (2006) Experimental Challenges to Theories of Classical Conditioning: Application of an Attentional Model of Storage and Retrieval Journal of Experimental Psychology 32, 1; 1-20.

[29]. Peters, R. S. (1960) The Concept of Motivation London: Routledge and Kegan Paul

[30]. Thompson, Richard F. (1985) The Brain: an Introduction to Neuroscience New York: W H. Freeman and Company, pp. 277, 292-293

[31]. Walker-Smith, J.A. Mittal, S.K. and Henderickse R.G. (1991) Disorders of the Digestive System in: Paediatrics in the Tropics (Eds.) Henderickse, R.G., Barr, D.G.D and Matthews T.S. Oxford: Blackwell Scientific Publications, p. 301 . 